\title{
Non-inertial effects on generalized KG-oscillator in the presence of Coulomb-type potential in topological defects geometry
}

Faizuddin Ahmed ( $\sim$ faizuddinahmed15@gmail.com )

National Academy Gauripur

\section{Research Article}

Keywords: cosmic string, relativistic wave-equations, energy spectrum, wave-functions, Aharonov-Bohm effect

Posted Date: February 1st, 2021

DOI: https://doi.org/10.21203/rs.3.rs-191273/v1

License: (a) (i) This work is licensed under a Creative Commons Attribution 4.0 International License.

Read Full License 


\title{
Non-inertial effects on generalized KG-oscillator in the presence of Coulomb-type potential in topological defects geometry
}

\author{
Faizuddin Ahmed ${ }^{1}$ \\ National Academy, Gauripur-783331, Assam, India
}

\begin{abstract}
In this work, we solve a generalized KG-oscillator subject to a scalar and vector potential of Coulomb-types under the effects of a uniform rotation in cosmic string space-time. We obtain the energy eigenvalue and eigenfunction, and analyze a relativistic analogue of the Aharonov-Bohm effect for bound states. We see that the presence of potential allow the formation of bound states solution and the energy level and wave-function for each radial mode depend on the global parameters of the space-time.
\end{abstract}

Keywords: cosmic string, relativistic wave-equations, energy spectrum, wave-functions, Aharonov-Bohm effect.

PACS Number(s): 03.65.Pm, 03.65.Ge, 98.80.Cq

\section{Introduction}

In relativistic quantum mechanics, the wave-equations have been of current research interest for theoretical physicists $[1,2,3]$. These wave-equations have investigated on the background curved geometries with or without interactions (e. g., $[4,5,6,7,8,9])$. In the context of cosmic string geometries, these wave-equations have studied by several authors (e. g.,[10, 11, 12, 13, 14, $15,16,17,18,19,20,21,22,23])$. In addition, the influence of non-inertial effects in cosmic string space-time was studied in [24, 25, 26, 27, 28, 29, 30, 31].

\footnotetext{
${ }^{1}$ faizuddinahmed15@gmail.com ; faiz4U.enter@rediffmail.com
} 
Cosmic string space-time in polar coordinates $(t, r, \Phi, z)$ is described by the following line element $[13,24,32,33,34]$ :

$$
d s^{2}=-d t^{2}+\alpha^{2} r^{2} d \Phi^{2}+d r^{2}+d z^{2}
$$

where $\alpha=(1-4 \mu)$ is the wedge parameter, and $\mu$ being the linear mass density of the string. The parameter $\alpha$ is related to the deficit angle $\nabla \phi=$ $2 \pi(1-\alpha)$. In cosmic string space-time, the parameter $\alpha$ values in the interval $0<\alpha<1$ within the general relativity. The line-element (1) describes a geometry with conical singularity which corresponds to the curvature tensor $R_{\rho, \Phi}^{\rho, \Phi}=\left(\frac{1-\alpha}{4 \alpha}\right) \delta^{2}(\vec{\rho})$, with $\delta^{2}(\vec{\rho})$ is the two-dimensional delta function. This conical singularity is such that the curvature is concentrated on the cosmic string axis, and in all other points, the curvature vanishes.

We do the following transformation into the metric (1) for a rotating reference frame as :

$$
\Phi=\phi+\omega t
$$

where $\omega>0$ is the constant angular velocity $[24,34,35,36]$ of the rotating frame. Inserting this transformation into the metric (1), we obtain the following line element:

$$
d s^{2}=-\left(1-\alpha^{2} \omega^{2} r^{2}\right) d t^{2}+2 \omega \alpha^{2} r^{2} d t d \phi+\alpha^{2} r^{2} d \phi^{2}+d r^{2}+d z^{2}
$$

An interesting feature of Eq. (3) is the condition

$$
0 \leq r<\frac{1}{\alpha \omega}
$$

This condition is related to the fact that for $r>\frac{1}{\alpha \omega}$ the velocity of the particle is greater than the speed of light. For this reason, it is convenient to restrict $r$ to the range $\left(0, \frac{1}{\alpha \omega}\right)$.

To obtain bound states solution of the relativistic wave-equation, various potentials have used in the relativistic quantum system. Among them much attention has given on Coulomb-like potential which is our interest here. This 
kind of potential has widely used by many authors (e. g., [37] and related references their in) and is given by

$$
S(r)=\frac{k_{c}}{r} \quad, \quad V(r)=\frac{\xi_{c}}{r} .
$$

where $k_{c}>0, \xi_{c}>0$ are the potential parameters. In this work, we have restricted that the scalar potential $S$ must be greater than the vector potential $V$, that is, $|S|>|V|$.

Inspired by the work [25], we solve a generalized KG-oscillator field in the presence of a scalar and vector potential of Coulomb-types with a magnetic quantum flux under non-inertial effects in cosmic string space-time, and analyze a relativistic analogue of the Aharonov-Bohm effect [38, 39].

\section{Generalized KG-oscillator in a rotating cos- mic string space-time with potentials}

The Klein-Gordon equation with modifying mass $M \rightarrow M+S(r)$ for a massive charged particle is described by [13]

$$
\left[\frac{1}{\sqrt{-g}} D_{\mu}\left(\sqrt{-g} g^{\mu \nu} D_{\nu}\right)-(M+S)^{2}\right] \Psi=0,
$$

where $D_{\mu}=\partial_{\mu}-i$ e $A_{\mu}$ is the minimal substitution. Here $e$ is the electric charge, $A_{\mu}$ is the four-vector potential of the electromagnetic field and $S$ is the scalar potential.

Now, let us assume that the topological defects (e.g., cosmic string) has an internal magnetic quantum flux field (with a magnetic flux $\Phi_{B}[40,41]$ ). We consider the four-vector potential

$$
A_{\mu}=(-V, \vec{A}) \quad, \quad A_{0}=V
$$

where the three vector gauge potential is defined by $\vec{A}=\left(0, A_{\phi}, 0\right)$ with the angular component $[34,35,36,42,43]$

$$
A_{\phi}=\frac{\Phi_{B}}{2 \pi} .
$$


Here $\Phi_{B}=$ const. is the internal magnetic quantum flux $[40,41]$ through the core of the topological defects [44]. It is worth mentioning that the AharonovBohm effect $[38,39]$ has investigated in the relativistic quantum mechanics (e. g., see $[45,36]$ and related references their in).

For the geometry (3), KG-equation (6) becomes

$$
\begin{aligned}
& {\left[-\left\{\left(\frac{\partial}{\partial t}+i e V\right)-\omega\left(\frac{\partial}{\partial \phi}-i e A_{\phi}\right)\right\}^{2}+\frac{1}{r} \frac{\partial}{\partial r}\left(r \frac{\partial}{\partial r}\right)+\frac{\partial^{2}}{\partial z^{2}}\right.} \\
& \left.+\frac{1}{\alpha^{2} r^{2}}\left(\frac{\partial}{\partial \phi}-i e A_{\phi}\right)^{2}-(M+S)^{2}\right] \Psi=0 .
\end{aligned}
$$

To couple the generalized oscillator with Klein-Gordon field, one can change the following generalized momentum operator [31, 45]

$$
\vec{p} \rightarrow \vec{p}-i M \Omega f(r) \hat{r} \quad \text { or } \quad \frac{\partial}{\partial r} \rightarrow \frac{\partial}{\partial r}+M \Omega f(r),
$$

where $\Omega$ is the oscillator frequency and $f(r)$ is the generalized function with $r$ being distance from the particle to the string. So we can write $p^{2} \rightarrow$ $(\vec{p}-i M \Omega f(r) \hat{r})(\vec{p}+i M \Omega f(r) \hat{r})$.

Therefore, generalized KG-oscillator equation becomes

$$
\begin{aligned}
& {\left[-\left\{\left(\frac{\partial}{\partial t}+i e V\right)-\omega\left(\frac{\partial}{\partial \phi}-i e A_{\phi}\right)\right\}^{2}+\frac{1}{r}\left(\frac{\partial}{\partial r}+M \Omega f(r)\right)\left(r \frac{\partial}{\partial r}-M \Omega r f(r)\right)\right.} \\
& \left.+\frac{\partial^{2}}{\partial z^{2}}+\frac{1}{\alpha^{2} r^{2}}\left(\frac{\partial}{\partial \phi}-i e A_{\phi}\right)^{2}-(M+S)^{2}\right] \Psi=0 .
\end{aligned}
$$

Since the metric is independent of time and symmetrical by translations along the $z$-axis, as well by rotations. It is reasonable to write down the solution to Eq. (11) as

$$
\Psi(t, r, \phi, z)=e^{i(-E t+l \phi+k z)} \psi(r),
$$

where $E$ is the energy of the particle, $l=0, \pm 1, \pm 2, \ldots$ are the eigenvalues of the $z$-component of the angular momentum operator, and $k$ is a constant. 
Substituting the solution (12) into the Eq. (11), we obtain the following radial wave-equation for $\psi(r)$ :

$$
\begin{aligned}
& \psi^{\prime \prime}(r)+\frac{1}{r} \psi^{\prime}(r)+\left[\left(E-e V+\omega\left(l-e A_{\phi}\right)\right)^{2}-(M+S)^{2}-k^{2}\right. \\
& \left.-\frac{\left(l-e A_{\phi}\right)^{2}}{\alpha^{2} r^{2}}-M \Omega\left(f^{\prime}(r)+\frac{f(r)}{r}\right)-M^{2} \Omega^{2} f^{2}(r)\right] \psi(r)=0 .
\end{aligned}
$$

Below we have chosen few arbitrary function $f(r)$, namely, Cornell-type and Coulomb-type potential form, respectively to study the generalized KGoscillator in the relativistic system.

\subsection{Cornell-type function}

In that case, we have chosen the function $f(r)=b_{1} r+\frac{b_{2}}{r}$, a Cornell-type potential form function where, $b_{1}>0, b_{2}>0$ are arbitrary constants. The first term is responsible for linear oscillator and second term leads to Coulomb oscillator.

Substituting potentials (5) and (8) into the Eq. (13) and using the above Cornell-type function, we obtain the following equation :

$$
\left[\frac{d^{2}}{d r^{2}}+\frac{1}{r} \frac{d}{d r}+\lambda-M^{2} \Omega^{2} b_{1}^{2} r^{2}-\frac{j^{2}}{r^{2}}-\frac{a}{r}\right] \psi(r)=0,
$$

where

$$
\begin{aligned}
\lambda & =\left(E+\alpha \omega l_{0}\right)^{2}-M^{2}-k^{2}-2 M \Omega b_{1}-2 M^{2} \Omega^{2} b_{1} b_{2}, \\
a & =2\left[e \xi_{c}\left(E+\alpha \omega l_{0}\right)+M k_{c}\right], \\
l_{0} & =\left(\frac{l-\Phi}{\alpha}\right)>0, \\
j & =\sqrt{l_{0}^{2}+M^{2} \Omega^{2} b_{2}^{2}+k_{c}^{2}-e^{2} \xi_{c}^{2}} .
\end{aligned}
$$

Since we have restricted $|S|>|V|$, so as to keep the parameter $j$ remain positive, we must have $\left|k_{c}\right|>\left|e \xi_{c}\right|$. 
Transforming $x=\sqrt{M \Omega b_{1}} r$ into the Eq. (14), we obtain the following equation:

$$
\left[\frac{d^{2}}{d x^{2}}+\frac{1}{x} \frac{d}{d x}+\zeta-x^{2}-\frac{j^{2}}{x^{2}}-\frac{\eta}{x}\right] \psi(x)=0,
$$

where

$$
\zeta=\frac{\lambda}{M \Omega b_{1}} \quad, \quad \eta=\frac{a}{\sqrt{M \Omega b_{1}}} .
$$

Now, we use the appropriate boundary conditions that the wave-functions is regular both at $x \rightarrow 0$ and $x \rightarrow \infty$. Suppose the possible solution to the Eq. (16) is

$$
\psi(x)=x^{j} e^{-\frac{x^{2}}{2}} H(x) .
$$

Substituting the solution (18) into the Eq. (16), we obtain the following equation

$$
H^{\prime \prime}(x)+\left[\frac{1+2 j}{x}-2 x\right] H^{\prime}(x)+\left[-\frac{\eta}{x}+\zeta-2-2 j\right] H(x)=0,
$$

Equation (19) is the biconfluent Heun's differential equation [13, 35, 46, 47] with $H(x)$ is the Heun polynomials function.

The above equation (19) can be solved by the Frobenius method. Writing the solution as a power series expansion around the origin [48]:

$$
H(x)=\sum_{i=0}^{\infty} c_{i} x^{i}
$$

Substituting the power series solution into the Eq. (19), we obtain the following recurrence relation

$$
c_{n+2}=\frac{1}{(n+2)(n+2+2 j)}\left[\eta c_{n+1}-(\zeta-2-2 j-2 n) c_{n}\right] .
$$

With few coefficients are

$$
\begin{aligned}
c_{1} & =\frac{\eta}{1+2 j} c_{0}, \\
c_{2} & =\frac{1}{4(1+j)}\left[\eta c_{1}-(\zeta-2-2 j) c_{0}\right] .
\end{aligned}
$$


The power series expansion $H(r)$ becomes a polynomial of degree $n$ by imposing the following two conditions $[6,7,9,13,45,35]$

$$
\begin{aligned}
\zeta-2-2 j & =2 n, \quad(n=1,2, \ldots) \\
c_{n+1} & =0 .
\end{aligned}
$$

By analyzing the first condition, we obtain following energy eigenvalue $E_{n, l}$ :

$$
\begin{aligned}
& E_{n, l}= \pm \sqrt{M^{2}+k^{2}+2 M \Omega b_{1}\left(n+2+\sqrt{\frac{(l-\Phi)^{2}}{\alpha^{2}}+M^{2} \Omega^{2} b_{2}^{2}+k_{c}^{2}-e^{2} \xi_{c}^{2}}\right)+2 M^{2} \Omega^{2} b_{1} b_{2}} \\
& -\omega|l-\Phi| .
\end{aligned}
$$

Note that Eq. (24) is not the general expression of the relativistic energy eigenvalue of a generalized KG-oscillator field in the presence of a magnetic quantum flux in rotating cosmic string space-time subject to a scalar and vector potentials of Coulomb-types. One can obtain the individual energy level and wave-function one by one by imposing the additional recurrence condition $c_{n+1}=0$ on the eigenvalue problem.

The corresponding wave-functions are given by

$$
\psi_{n, l}(x)=x^{\sqrt{\frac{(l-\Phi)^{2}}{\alpha^{2}}+M^{2} \Omega^{2} b_{2}^{2}+k_{c}^{2}-e^{2} \xi_{c}^{2}}} e^{-\frac{x^{2}}{2}} H(x) .
$$

Now, we evaluate the individual energy level and eigenfunction one by one as in $[13,24,35,34]$. For example, $n=1$, we have $\zeta-2-2 j=2$ and $c_{2}=0$ which implies

$$
\begin{aligned}
& \Rightarrow \frac{\eta}{1+2 j}=\frac{2}{\eta} \\
& \Rightarrow \Omega_{1, l}=\left[\frac{a_{1, l}^{2}}{2 M b_{1}(1+2 j)}\right]
\end{aligned}
$$

a constraint on the oscillator frequency $\Omega_{1, l}$. Note that its values changes for each quantum number $n$ and $l$, so we have labeled $\Omega \rightarrow \Omega_{n, l}$ and $a \rightarrow a_{n, l}$ 
since it relates with energy $E_{n, l}$ given by Eq. (15). We can note from Eq. (26) that the possible values of the frequency depend on the quantum numbers which shows a quantum effect. In addition, for each relativistic energy level, we have a different relation of the oscillator frequency associated to scalar and vector potentials, and of the quantum numbers of the relativistic system.

Since the frequency parameter $\Omega_{1, l}$ is related with $a_{1, l}$ which is already related with $E_{1, l}$ by Eq. (15). Therefore, the ground state energy level $E_{1, l}$ can be obtained from the following expression

$$
\begin{aligned}
E_{1, l}= & \pm \sqrt{M^{2}+k^{2}+2 M \Omega_{1, l} b_{1}\left(3+\sqrt{\frac{(l-\Phi)^{2}}{\alpha^{2}}+M^{2} \Omega_{1, l}^{2} b_{2}^{2}+k_{c}^{2}-e^{2} \xi_{c}^{2}}\right)+2 M^{2} \Omega_{1, l}^{2} b_{1} b_{2}} \\
& -\omega|l-\Phi| .
\end{aligned}
$$

And the ground state eigenfunction

$$
\psi_{1, l}(x)=x^{\sqrt{\frac{(l-\Phi)^{2}}{\alpha^{2}}+M^{2} \Omega_{1, l}^{2} b_{2}^{2}+k_{c}^{2}-e^{2} \xi_{c}^{2}}} e^{-\frac{x^{2}}{2}}\left(c_{0}+c_{1} x\right)
$$

where

$$
c_{1}=\sqrt{\frac{2}{\left(1+2 \sqrt{\frac{(l-\Phi)^{2}}{\alpha^{2}}+M^{2} \Omega_{1, l}^{2} b_{2}^{2}+k_{c}^{2}-e^{2} \xi_{c}^{2}}\right)}} c_{0} .
$$

We can see that the lowest energy state is defined by (27) plus the expression given in (28)-(30) for the radial mode $n=1$, instead of $n=0$. This effect arises due to the presence of Coulomb-types potential in the relativistic quantum system.

We have seen that the relativistic energy eigenvalue and wave-function depends on the geometric quantum phase. Thus, we have that, $E_{n, l}\left(\Phi_{B}+\right.$ $\left.\Phi_{0}\right)=E_{n, l \mp \tau}\left(\Phi_{B}\right)$ where, $\Phi_{0}= \pm \frac{2 \pi}{e} \tau$ with $\tau=0,1,2, \ldots$ This dependence of the relativistic energy level on the geometric quantum phase gives rise to a relativistic analogue of the Aharonov-Bohm effect for bound states. 


\subsection{Coulomb-type function}

We have chosen the function $f(r)=\frac{b_{2}}{r}$ in the considered quantum system which is called Coulomb oscillator. Therefore Eq. (14) becomes

$$
\left[\frac{d^{2}}{d r^{2}}+\frac{1}{r} \frac{d}{d r}+\tilde{\lambda}-\frac{j^{2}}{r^{2}}-\frac{a}{r}\right] \psi(r)=0,
$$

where

$$
\tilde{\lambda}=\left(E+\alpha \omega l_{0}\right)^{2}-M^{2}-k^{2} .
$$

The above equation (30) can be transformed as [49]

$$
\left[\frac{d^{2}}{d r^{2}}+\frac{1}{r} \frac{d}{d r}+\frac{1}{r^{2}}\left(-\xi_{1} r^{2}+\xi_{2} r-\xi_{3}\right)\right] \psi(r)=0
$$

where

$$
\xi_{1}=-\tilde{\lambda} \quad, \quad \xi_{2}=-a \quad, \quad \xi_{3}=j^{2} .
$$

Therefore, the energy eigenvalue expression is given by

$$
\begin{aligned}
E_{n^{\prime}, l}= & \frac{1}{\left[\left(n^{\prime}+\frac{1}{2}+j\right)^{2}-e^{2} \xi_{c}^{2}\right]} \times \\
& {\left[e M \xi_{c} k_{c} \pm\left(n^{\prime}+\frac{1}{2}+j\right) \sqrt{\left(M^{2}+k^{2}\right)\left\{\left(n^{\prime}+\frac{1}{2}+j\right)^{2}-e^{2} \xi_{c}^{2}\right\}-M^{2} k_{c}^{2}}\right] } \\
& -\omega|l-\Phi|,
\end{aligned}
$$

where $n^{\prime}=0,1,2,3, \ldots$ and $j=\sqrt{\frac{(l-\Phi)^{2}}{\alpha^{2}}+M^{2} \Omega^{2} b_{2}^{2}+k_{c}^{2}-e^{2} \xi_{c}^{2}}$. The corresponding eigenfunction is given by

$$
\psi_{n^{\prime}, l}(r)=|N|_{n^{\prime}, l} r \sqrt{\frac{(l-\Phi)^{2}}{\alpha^{2}}+M^{2} \Omega^{2} b_{2}^{2}+k_{c}^{2}-e^{2} \xi_{c}^{2}} e^{-\frac{r}{2}} L_{n^{\prime}}^{\left(\sqrt{\left.\frac{(l-\Phi)^{2}}{\alpha^{2}}+M^{2} \Omega^{2} b_{2}^{2}+k_{c}^{2}-e^{2} \xi_{c}^{2}\right)}\right.}(r),
$$

where $N_{n^{\prime}, l}=\left(\frac{n^{\prime} !}{\left(n^{\prime}+\frac{|l-\Phi|}{\alpha}\right) !}\right)^{\frac{1}{2}}$ is the normalization constant and $L_{n^{\prime}}^{\left(\sqrt{\frac{(l-\Phi)^{2}}{\alpha^{2}}+M^{2} \Omega^{2}+k_{c}^{2}-e^{2} \xi_{c}^{2}}\right)}(r)$ is the generalized Laguerre polynomials and are orthogonal over $[0, \infty)$ with respect to the measure with weighting function $r^{j} \mathrm{e}^{-r}$ as

$$
\int_{0}^{\infty} r^{j} e^{-r} L_{n^{\prime}}^{(j)} L_{m^{\prime}}^{(j)} d r=\frac{\left(n^{\prime}+j\right) !}{n^{\prime} !} \delta_{n^{\prime} m^{\prime}}
$$


Here also, we have seen that the relativistic energy level depends on the geometric quantum phase. Thus, we have that, $E_{n^{\prime}, l}\left(\Phi_{B}+\Phi_{0}\right)=E_{n^{\prime}, l \mp \tau}\left(\Phi_{B}\right)$ where, $\Phi_{0}= \pm \frac{2 \pi}{e} \tau$ with $\tau=0,1,2, \ldots$. This dependence of the relativistic energy eigenvalues on the geometric quantum phase gives rise to a relativistic analogue of the Aharonov-Bohm effect.

\section{Conclusions}

In this paper, we have solved a generalized Klein-Gordon oscillator by changing the momentum operator $\vec{p} \rightarrow \vec{p}-i M \Omega f(r) \hat{r}$ where, $f(r)$ is an arbitrary function subject to a scalar and vector potentials of Coulomb-types including a magnetic flux in the rotating cosmic string space-time. In sub-section 2.1, we have chosen a Cornell-type function $f(r)$ and obtained the relativistic energy spectrum Eq. (24) and wave-functions by Eq. (25). By imposing the recurrence condition $c_{n+1}=0$ on the eigenvalue problem, we have obtained the individual energy levels and eiegnfunction, for example $n=1$, and others are in the same way. In sub-section 2.2, we have chosen a Coulomb-type function $f(r)$ and obtained the relativistic energy spectrum Eq. (34) and wave-functions by Eq. (35) using the Nikiforov-Uvarov method. We have seen that the energy eigenvalues get modify in comparison to those in [25] due to the presence of Coulomb-type potentials, and the magnetic quantum

flux. Thus the presence of Coulomb-type potential allow the formation of bound states and the energy spectrum associated with the oscillator field depends on the magnetic quantum flux $\Phi_{B}$ which gives rise to a relativistic analogue of the Aharonov-Bohm effect.

An important result that we have seen is the non-inertial effect that restricts the region of space-time. So there exist a coupling between the angular quantum number $l$ and the angular velocity $\omega$ of the rotating frame which gives rise to a Sagnac-type effect $[50,51,52]$. 


\section{Conflict of Interests}

Author declares that there is no conflict of interests regarding publication this paper.

\section{Data Availability}

There is no data associated with this manuscript.

\section{References}

[1] B. Thaller, The Dirac Equation, Springer, New York (1992).

[2] A. W. Thomas and W. Weise, Structure of the Nucleon, Wiley, Berlin (2001).

[3] T. Y. Wu and W. Y. P. Hwang, Relativistic Quantum Mechanics and Quantum Fields, World Scientific Publishing Co. Pvt. Ltd., Singapore (1991).

[4] B. D. B. Figueiredo, I. D. Soares and J. Tiomno, Class. Quantum Grav. 9, 1593 (1992).

[5] J. Carvalho, A. M. de M. Carvalho and C. Furtado, Eur. Phys. J. C (2014) $74: 2935$.

[6] Z. Wang, Z. Long, C. Long and M. Wu, Euro. Phys. J. Plus (2015) 130 : 36 .

[7] R. L. L. Vitória, C. Furtado and K. Bakke, Eur. Phys. J. C (2018) 78 : 44.

[8] M. Eshghi and M. Hamzavi, Eur. Phys. J. C (2018) 78 : 522.

[9] R. L. L. Vitória and K. Bakke, Euro. Phys. J. Plus (2018) 133 : 490. 
[10] A. Boumali and N. Messai, Can. J. Phys. 92, 1460 (2014).

[11] K. Bakke and C. Furtado, Ann. Phys. (N. Y.) 355, 48 (2015).

[12] R. L. L. Vitória, C. Furtado and K. Bakke, Ann. Phys. (N. Y.) 370, 128 (2016).

[13] E. R. Figueiredo Medeiros and E. R. Bezerra de Mello, Eur. Phys. J. C (2012) $72: 2051$.

[14] M. Hosseini, H. Hassanabadi, S. Hassanabadi and P. Sedaghatnia, Int. J. Geom. Meths. Mod. Phys. (2019) 16 : 1950054.

[15] M. Hosseinpour, H. Hassanabadi and F. M. Andrade, Eur. Phys. J. C (2018) $78: 93$.

[16] H Hassanabadi, M Hosseinpour and M de Montigny, Eur. Phys. J. Plus (2017) $132: 541$.

[17] M. Hosseinpour, F. M. Andrade, E. O. Silva and H. Hassanabadi, Eur. Phys. J. C (2017) $77: 270$.

[18] M. Hosseinpour and H. Hassanabadi, Int. J. Mod. Phys. A 30, 1550124 (2015).

[19] M. de Montigny, M. Hosseinpour and H. Hassanabadi, Int. J. Mod. Phys. A 31, 1650191 (2016).

[20] L. B. Castro, Eur. Phys. J. C (2015) 75 : 1.

[21] H. Sobhani, H. Hassanabdi and W. S. Chung, Eur. Phys. J. C (2018) $78: 106$.

[22] K. Bakke, Eur. Phys. J. Plus (2012) $127: 82$.

[23] K. Bakke and C. Furtado, Eur. Phys. J. C (2010) 69 : 531. 
[24] K. Bakke, Gen. Relativ. Grav. (2013) $45: 1847$.

[25] L. C. N. Santos and C. C. Barros Jr., Eur. Phys. J. C (2018) 78 : 13.

[26] M. Hosseinpour, H. Hassanabadi and M. de Montigny, Int. J. Geom. Meths. Mod. Phys. (2019) $15: 1850165$.

[27] M. Hosseinpour and H. Hassanabadi, Adv. High Energy Phys. 2018, 2959354 (2018).

[28] M. Hosseinpour, H. Hassanabadi and M. de Montigny, Eur. Phys. J. C (2019) $79: 311$.

[29] B. -Q. Wang, Z. -W. Long, C.-Y. Long and S.-R. Wu, Mod. Phys. Lett. A 33, 1850025 (2018).

[30] C. R. Muniz, V. B. Bezerra and M. S. Cunha, Ann. Phys. 350, 105 (2014).

[31] S. Zare, H. Hassanabadi and M. de Montigny, Gen. Relativ. Grav. (2020) $52: 25$.

[32] A. Vilenkin, Phys. Lett. B 133, 177 (1983).

[33] A. Vilenkin, Phys. Rep. 121, 263 (1985).

[34] F. Ahmed, Int. J. Geom. Meths. Mod. Phys. 17, 2050138 (2020).

[35] F. Ahmed, Chin. J. Phys. 66, 587 (2020).

[36] F. Ahmed, EPL 131, 30002 (2020).

[37] M. K. Bahar and F. Yasuk, Adv. High Energy Phys. 2013, 814985 (2013).

[38] M. Peshkin and A. Tonomura, Lect. Notes Phys. 340, Springer-Verlag, Berlin, Germany (1989). 
[39] V. B. Bezerra, J. Math. Phys. 30, 2895 (1989).

[40] Y. Aharonov and D. Bohm, Phys. Rev. 115, 485 (1959).

[41] G. de A. Marques, C. Furtado, V. B. Bezerra and F. Moraes, J. Phys. A 34, 5945 (2001).

[42] R. L. L. Vitória and K. Bakke, Euro. Phys. J. Plus (2016) 131 : 36.

[43] M. S. Cunha, C. R. Muniz, H. R. Christiansen and V. B. Bezerra, Euro. Phys. J. C (2016) 76 : 512.

[44] C. Furtado, F. Moraes and V. Bezerra, Phys. Rev. D 59, 107504 (1999).

[45] F. Ahmed, EPL 130, 40003 (2020).

[46] A. Ronveaux, Heun's Differential Equations, Oxford University Press, Oxford ( 1995).

[47] S. Y. Slavyanov and W. Lay, Special Functions: A Unified Theory Based in Singularities, Oxford University Press, New York (2000).

[48] G. B. Arfken and H. J. Weber, Mathematical Methods For Physicists, Elsevier Academic Press, London (2005).

[49] A. F. Nikiforov and V. B. Uvarov, Special Functions of Mathematical Physics, Birkhauser, Basel (1988).

[50] M. G. Sagnac, C. R. Acad. Sci. 157, 708 (1913).

[51] M. G. Sagnac, C. R. Acad. Sci. (Paris) 157, 1410 (1913).

[52] F. Hehl and W. -T. Ni, Phys. Rev. D 42, 2045 (1990). 